\title{
Incidence of medication errors in a surgical intensive care unit. analisys and improvement measures implemented
}

\author{
P Cuesta-Montero ${ }^{1 *}$, E Domingo-Chiva², S Plata-Paniagua², JA Monsalve- Naharro', B Montalban-Moreno', \\ JM Jimenez-Vizuete', A Valladolid-Walsh', R Peyro-García'
}

From ESICM LIVES 2015

Berlin, Germany. 3-7 October 2015

\section{Introduction}

Medication errors in critical care are frequent, serious and predictable. Critically ill patients are prescribed twice as many medications as patients outside the intensive care unit and nearly all will suffer a potential error at some point during their stay.

\section{Objectives}

To quantify and characterize medication errors in a surgical intensive care unit (SICU).

\section{Methods}

We conducted a one-month prospective observational study to detect, quantify and score medication errors in a SICU.

\section{Results}

A total of 634 observations made over weekdays and weekends were performed including morning, noon and night shifts. $36.27 \%$ observations (230) included some type of error, a total of 245 medication errors were detected. According to the type of error found: 52 were prescription errors (21.22\%), 2 omissions (0.82\%), 44 related to administration technique (wrong speed) (17.96\%), 10 omissions of the administration record (4.08\%), 97 erroneous preparations $(39.59 \%), 1$ wrongly prescribed dose by default $(0.41 \%)$ and 3 by excess (1.22\%), 5 errors related to erroneous administration route (2.04\%), 2 erroneous drug monitorization $(0.82 \%)$ and 29 transcription errors (11.84\%). According to severity within categories established by the National Coordinating Council for Medication
Error Reporting and Prevention (NCCMERP), 26.12\% errors were Category A, $10.20 \%$ were Category B, $61.63 \%$ were Category C, $1.63 \%$ Category D and $0.41 \%$ Category F.

\section{Conclusions}

Determining the incidence of medication errors in our system and adopting measures to prevent them is a priority in order to improve the drug treatment process in critically ill patients. The integration of a pharmacist in the intensive care unit allowed us to implement improvement measures to reduce medication related errors and improve quality of care. Measures implemented included: - Development of pharmacotherapeutic protocols and guidelines drug management. - Conducting training sessions for medical staff and nursing.

\section{Authors' details}

${ }^{1}$ Complejo Hospitalario Universitario de Albacete, Anesthesiology and Critical Care, Albacete, Spain. ${ }^{2}$ Complejo Hospitalario Universitario de Albacete, Pharmacy, Albacete, Spain.

Published: 1 October 2015

\section{Reference}

1. Calabrese AD: Intensive Care Med 2001, 27:1592-8. 